\title{
Papaverine and hepatotoxicity
}

\author{
M. S. PathY* \\ F.R.C.P., F.R.C.P.(E) \\ A. J. ReYnOldS \\ M.B. B.S. \\ Departments of *Geriatric Medicine, and of Dermatology, University Hospital of Wales, Cardiff
}

\begin{abstract}
Summary
Six of 14 patients receiving a sustained release papaverine preparation developed abnormal liver function tests. One patient had jaundice and another abnormal liver histology on a biopsy specimen. This is only the third report of impaired liver function following the administration of papaverine-a drug which has been used in clinical medicine for over 100 years.
\end{abstract}

\section{Introduction}

Papaverine is an alkaloid of the benzylisoquinoline group and has been widely used as a smooth muscle relaxant for more than a century. It decreases cerebrovascular resistance and papaverine hydrochloride has been much used in the U.S.A. in recent years in patients with cerebral arterial disease and peripheral arterial disease (Jayne, Scheinberg and Belle, 1952; Whittier, 1964; Stern, 1970). Side effects following oral administration were considered insignificant and largely limited to nausea, vomiting or throbbing headache. Hepatotoxic sequelae to papaverine administration were first reported in 4 elderly patients (Rønnov-Jessen and Tjerlund, 1969). An increase in serum alkaline phosphatase levels may occur in up to $43 \%$ of older people on daily papaverine (Dreimen, 1973), although this has been refuted (Gillis, 1973).

\section{Patients and methods}

A double-blind placebo controlled trial of the sustained release form of papaverine hydrochloride -Pavigrad-was undertaken to assess its effect on intellectual impairment in a group of elderly patients with clinical evidence of focal vascular brain damage, i.e. hemiplegia. The total daily dose of papaverine was $600 \mathrm{mg}$. During the trial, 14 patients received Pavigrad for up to 12 weeks. No other drug therapy was administered during the trial period.

\section{Results}

All but one of the 14 patients had normal liver function tests before drug administration. Of the 14,7 had no adverse reaction or change in liver

Reprint requests to Dr M. S. Pathy. function tests. Of the remainder, the drug was discontinued in one patient after 2 weeks owing to nausea, vomiting and abdominal pain, but, unfortunately, the liver function tests were not documented. After 2-12 weeks on papaverine, 3 patients had mild elevations of serum alkaline phosphatase levels ranging from 48-57 i.u./l (normal, 10-40) and in one patient the $\gamma$-glutamyl transpeptidase $(\gamma-G T)$ rose to 69 i.u./l (normal, <45).

The remaining 3 patients had more marked abnormalities:

\section{Patient no. 1}

After 3 weeks on Pavigrad he complained of nausea and developed jaundice the following day. The bilirubin rose to $74 \mu \mathrm{mol} / \mathrm{l}$ (normal, <17), the aspartate aminotransferase (AST) to 130 i.u. (normal, 10-40), alkaline phosphatase to 130 i.u./l, and $\gamma$-GT to 343 i.u./l. The drug was discontinued and the jaundice subsided and liver function tests reverted to normal.

\section{Patient no. 2}

A 72-year-old female who had a slight pre-trial elevation of the alkaline phosphatase at 47 i.u./l, after taking Pavigrad for 5 weeks, reported that her urine was dark. The liver function tests were as follows: bilirubin, $38 \mu \mathrm{mol} / \mathrm{l}$; AST, 69 i.u./l; alkaline phosphatase, 190 i.u./l; and $\gamma$-GT, 1080 i.u./l. The drug was discontinued. Although the liver function tests started to improve, she developed a myocardial infarction and severe congestive heart failure in week 9. A liver biopsy showed the liver architecture to be normal and there was no evidence of cholestasis or cell necrosis (Fig. 1). There was a moderate non-specific chronic inflammatory infiltration in the portal tracts consisting of lymphocytes and a few macrophages but no eosinophils. One portal tract showed a non-caseating granuloma (Z.N.-negative). The Kupffer cells were increased.

\section{Patient no. 3}

A male aged 72 years who had vomiting and intermittent abdominal pain in Week 12. Liver function tests performed were as follows: bilirubin, $18 \mu \mathrm{mol} / 1$; 


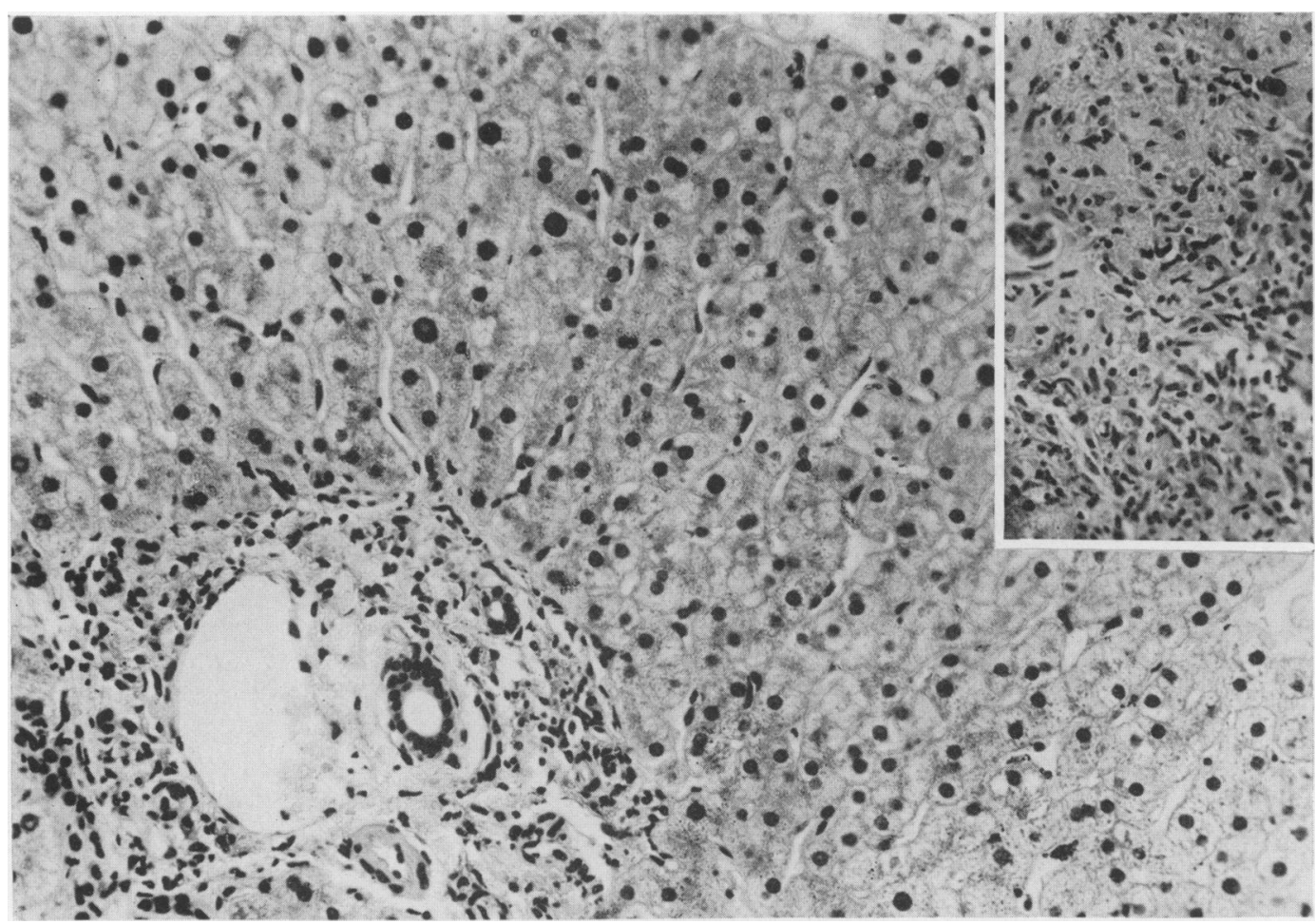

FIG. 1. Liver showing non-specific portal chronic inflammatory infiltrate. Kupffer cells are prominent. The inset shows part of a granuloma from another portal tract $(\mathrm{HE}, \times 216)$.

AST 92 i.u./1; alkaline phosphatase 59 i.u./l; and $\gamma$-GT, 312 i.u./l. Papaverine was discontinued and the tests returned to normal within 4 weeks.

\section{Discussions and conclusions}

The hepatotoxicity of papaverine was first noted in 4 patients by Rønnov-Jessen and Tjerlund (1969). In a later study, 13 out of 30 elderly patients were found to have developed an elevated serum alkaline phosphatase after the oral administration of sustained release papaverine (Driemen, 1973); but in another report no clinical or biochemical evidence of hepatotoxicity could be ascertained in 29 elderly patients receiving $600 \mathrm{mg}$ of Pavigrad daily for 19 weeks (Gillis, 1973).

Rønnov-Jessen and Tjerlund (1969) and Kiaer, Olsen and Rønnov-Jessen (1974) reported the liver histology characteristically to show diffuse proliferation of the Kupffer cells and infiltration of the portal spaces with eosinophilic granulocytes, although the latter finding was absent in one of their patients. Kiaer et al. (1974) gave repeated courses of papaverine with production of recurrent evidence of hepatoxicity to some of their patients and included in their study 2 patients originally reported by
Rønnov-Jessen and Tjerlund. They found that all patients exposed anew produced prompt reactions. They consider that this finding and the presence of eosinophilic infiltration of the portal spaces indicated that the hepatotoxic action of papaverine includes an allergic factor. Zimmerman (1969) suggested that papaverine-induced liver injury might be due to a combination of allergy to the drugs and to a metabolic aberration of the host permitting the accumulation of hepatotoxic metabolites.

Changes in liver function tests occurred in the authors' patients after a latent period of 2-12 weeks which is comparable to the 3-6 weeks in the series of Kiaer et al. (1974). All authors have noted a rapid return of liver function tests to normal on discontinuing the drug. This finding was also noted in the present patients with the exception of patient no. 2 who developed severe congestive heart failure following a myocardial infarction at week 9. Six of the 14 subjects in the present study had biochemical evidence of altered hepatic function, the most consistent changes being an elevation in the serum alkaline phosphatase or $\gamma-\mathrm{GT}$, typical of a portal infiltrate or granulomata, although the elevated AST levels in some patients suggest hepatocellular 
damage. The absence of eosinophils in the present biopsy compared to other published cases may be due to the fact that the specimen was taken after discontinuation of the drug.

Mild to moderately severe changes in liver function tests in $50 \%$ of a carefully supervised group of elderly patients receiving papaverine suggests that this drug is inappropriate for the elderly. A trial of papaverine in intellectually impaired patients with cerebro-vascular disease was accordingly abandoned.

\section{Acknowledgments}

We are grateful to Dr J. Gough, Consultant Pathologist, Department of Pathology, Welsh National School of Medicine, Cardiff, for his report on the liver histology, and to Mr P. Langham for the photomicrograph.

\section{References}

DrIemen, P.M. (1973) Papaverine-hepatotoxicity or not? Journal of the American Geriatrics Society. 21, 202.

GiLlis, M.R. (1973) Papaverine-safety in use. Journal of the American Geriatrics Society, 21, 200.

JAYNe, H.W., ScheinberG, M.R. \& Belle, M.S. (1952) The effect of intravenous papaverine hydrochloride on the cerebral circulation. Journal of Clinical Investigation, 31, 111.

Rønnov-Jessen, V. \& Tuerlund, A. (1969) Hepatotoxicity due to treatment with papaverine. New England Journal of Medicine, 281, 1333.

STERN, F.H. (1970) Management of chronic brain syndrome secondary to cerebral arteriosclerosis with special reference to papaverine hydrochloride. Journal of the American Geriatrics Society, 18, 507.

WhitTIER, J.R. (1964) Vasorelaxant drugs and cerebro vascular disease. Angiology, 15, 82.

Zimmerman, H.J. (1969) Papaverine revisited as a hepatotoxin. New England Journal of Medicine, 281, 1364. 